\title{
NEDD4 promotes cell growth and migration via PTEN/PI3K/AKT signaling in hepatocellular carcinoma
}

\author{
ZHI-JUN HUANG ${ }^{1}$, JUN-JUN ZHU ${ }^{2}$, XIAO-YU YANG ${ }^{3}$ and EWELINA BISKUP ${ }^{4}$ \\ ${ }^{1}$ Department of General Surgery, First People's Hospital of Yancheng, Yancheng, Jiangsu 224005; \\ ${ }^{2}$ Department of Radioactive Intervention, ${ }^{3}$ Second Department of Special Treatment, Eastern Hepatobiliary Surgery Hospital, \\ Second Military Medical University, Shanghai 200438, P.R. China; ${ }^{4}$ Department of Oncology, \\ University Hospital of Basel, 4055 Basel, Switzerland
}

Received December 3, 2015; Accepted January 31, 2017

DOI: $10.3892 / \mathrm{ol} .2017 .6532$

\begin{abstract}
ThenovelE3 ubiquitin-protein ligaseneuralprecursor cell-expressed developmentally downregulated protein 4 (NEDD4) has been implicated as a crucial factor promoting the tumorigenesis of several types of cancer. The present study investigated the oncogenic role of NEDD4 in hepatocellular carcinoma (HCC) by targeted small interfering RNA silencing of the tumor suppressor phosphatase and tensin homolog (PTEN). Using normal hepatocyte and HCC cell lines, the influence of NEDD4 depletion on proliferation and migration as well as on the PTEN/phosphatidylinositol-3-kinase/protein kinase B signaling pathway was assessed. Additionally, the expression of NEDD4 was assessed in HCC specimens from 78 patients. The in vitro immunohistochemistry results indicated that NEDD4 protein expression was higher, but PTEN expression was lower, in HCC cells compared with normal hepatocytes. The results from the MTT assay, wound healing experiment and Transwell assays demonstrated that NEDD4 depletion lead to decreased proliferation and migration ability of HCC cells. Results from western blotting and immunofluorescence demonstrated that silencing of NEDD4 disrupted the PTEN/phosphoinositide 3-kinase (PI3K)/protein kinase B (AKT) signaling pathway in HCC cells. A total of 55 (70.5\%) of the HCC specimens stained positive for NEDD4 and expression significantly correlated with tumor size $(\mathrm{P}=0.047)$, differentiation degree $(\mathrm{P}=0.032)$, vascular invasion $(\mathrm{P}<0.001)$, and lymph node metastasis $(\mathrm{P}=0.005)$. Thus, NEDD4 appears to perform a critical role in promoting the proliferation and
\end{abstract}

Correspondence to: Dr Xiao-Yu Yang, Second Department of Special Treatment, Eastern Hepatobiliary Surgery Hospital, Second Military Medical University, 225 Changhai Road, Shanghai 200438, P.R. China

E-mail: ehbh_yangxy@163.com

Key words: hepatocellular carcinoma, neural precursor cell-expressed developmentally downregulated protein 4, phosphatase and tensin homolog, phosphatidylinositol-3-kinase/protein kinase B, cell growth, metastasis, tumorigenesis, cell migration metastasis of HCC via activation of the PTEN/PI3K/AKT signaling pathway; as such, NEDD4 may be a promising target for novel treatments of $\mathrm{HCC}$.

\section{Introduction}

Hepatocellular carcinoma (HCC) is the fifth most common type of malignancy worldwide, and its incidence rate is increasing (1-3). Although intensive studies have improved early diagnosis and therapeutic options, providing minimally invasive approaches for hepatic resection and liver transplantation, the overall prognosis of HCC remains poor due to high rates of metastasis and recurrence $(4,5)$. The accompanying high rate of mortality for metastatic HCC presents an urgent need to identify key molecules that can be used as prognostic and diagnostic markers to further improve early detection, as well as approaches towards prevention, antagonizing metastatic progression and therapy.

The neural precursor cell-expressed developmentally downregulated gene 4 (NEDD4, also termed NEDD4-1) is a novel E3 ubiquitin-protein ligase that targets proteins for ubiquitination and degradation. In addition, NEDD4 regulates a large number of membrane proteins, including ion channels and membrane receptors via its ubiquitination activities and ability to mediate endocytosis (6). Ubiquitination is crucial for controlling the turnover rate, localization and activity of cellular proteins (7). While NEDD4 was initially identified as a critical regulator of neuronal function and plasticity in the brain (8), other studies have shown that NEDD4 also performs a role in tumorigenesis and cancer development. Specifically, aberrant expression of NEDD4 was shown to lead to malignant transformation $(9,10)$. Additionally, a further study into the liver revealed an important regulatory role for NEDD4 in tissue regeneration (11). The phosphoinositide 3-kinase (PI3K)-protein kinase B (AKT) signaling pathway is hyper-activated in the majority of cancers. Phosphatase and tensin homolog deleted on chromosome 10 (PTEN) is a central negative regulator of PI3K-Akt signal transduction by dephosphorylating phosphoinositide (PI) (3-5), P3 and inhibiting downstream signals (12).

The function of NEDD4 in HCC and its interactions with the tumor suppressor PTEN remain to be elucidated. Therefore, 
the present study aimed to investigate the function of NEDD4 in $\mathrm{HCC}$ in relation to PTEN and PI3K/AKT signaling. The results provide additional information regarding the role of NEDD4 as an oncogene in HCC and its potential as a diagnostic and therapeutic target.

\section{Materials and methods}

Patients and tissue specimens. Hepatic surgical specimens of HCC and matched adjacent non-tumorous tissues were collected from 78 patients who underwent curative surgery between April 2007 and July 2012 at the First People's Hospital of Yancheng (Yancheng, China). All specimens were fixed in formalin, embedded in paraffin and sectioned for analysis. All patients had histologically-confirmed HCC. None of the patients had undergone preoperative interventions, including neo-adjuvant radio- or chemotherapy, percutaneous ablation or chemo-embolization. The Ethics Committee of the First People's Hospital of Yancheng approved the study protocol. Written informed consent was obtained from every patient preoperatively, and study participation was voluntary. The clinical baseline characteristics of the HCC patients are presented in Table I.

Cell culture. The human HCC Huh7, Hep3B, PLC/PRF/5 and SMMC7721 cell lines, and the human normal liver cell line LO2 were purchased from the Cell Bank of the Chinese Academy of Science (Shanghai, China). All cells were cultured in DMEM medium (Gibco; Thermo Fisher Scientific, Inc., Waltham, MA, USA) containing 10\% fetal bovine serum (Gibco; Thermo Fisher Scientific, Inc.), $100 \mathrm{U} / \mathrm{ml}$ penicillin and $100 \mathrm{U} / \mathrm{ml}$ streptomycin (Invitrogen; Thermo Fisher Scientific, Inc.), and maintained in a $5 \% \mathrm{CO}_{2}$-humidified atmosphere at $37^{\circ} \mathrm{C}$.

Small interfering (si)RNA. The Hep3B cells that overexpress NEDD4 were selected for siRNA experiments. Briefly, the cells were seeded $\left(5 \times 10^{5}\right.$ cells/well) in 6 -well plates and grown at $37^{\circ} \mathrm{C}$ for $24 \mathrm{~h}$ to $60-80 \%$ confluence. Transient-transfection was carried out with NEDD4-targeting siRNA sequence (5'-UUCAAUUGCCAUCUGAAGUUUAUCC-3'; Thermo Fisher Scientific, Inc.) or non-targeting siRNA (5'-CCGGAU UUAAAGCCGAAACCCGGUU-3') as a control (13) using the previously determined optimal concentration $(2 \mu \mathrm{g} / \mathrm{well}$ respectively) and Lipofectamine ${ }^{\circledR} 2000$ for $48 \mathrm{~h}$ at $37^{\circ} \mathrm{C}$ (Thermo Fisher Scientific, Inc.).

Cell viability assay. Cellular viability was assessed by the MTT assay. Briefly, $5 \times 10^{3}$ cells/well were plated in 96-well plates, incubated at $37^{\circ} \mathrm{C}$ overnight, and transfected with NEDD4-siRNA or non-targeting siRNA. Following 24 and $48 \mathrm{~h}$ of incubation, $20 \mu \mathrm{l}$ of MTT $(5 \mathrm{mg} / \mathrm{ml})$ was added to each well. Subsequent to an additional $4 \mathrm{~h}$ of incubation, $150 \mu 1$ of dimethyl sulfoxide was added to each well and optical density values were measured at $570 \mathrm{~nm}$ (14).

Wound healing and invasion assays. Cells were seeded at $4 \times 10^{5}$ cells/well in 6-well plates and incubated for $24 \mathrm{~h}$ at $37^{\circ} \mathrm{C}$. A wound was made by scratching a straight line in the monolayer of cells and NEDD4-siRNA transfection was carried out. At 0,24 and $48 \mathrm{~h}$, dead cells were removed by washing with
PBS and the migration ability was observed using an Olympus BX61 upright microscope equipped with imaging technology (Olympus Corporation, Tokyo, Japan). Migration distance was measured and migration area was calculated automatically by using the Image J version 1.42 software (National Institutes of Health, Bethesda, MA, USA).

Cell invasion assays were carried out using the Transwell chamber assay with Matrigel (EMD Millipore, Billerica, MA, USA) (14). Matrigel (Sigma-Aldrich; Merck KGaA) was added to the filter to form a thin gel layer. Following incubation at $37^{\circ} \mathrm{C}$ for $48 \mathrm{~h}$, the cells adherent to the upper surface of the filter were removed using a cotton applicator, then stained with crystal violet. Ten random fields were selected and the values obtained were calculated by averaging the total numbers of cells from triplicate determinations.

Western blot analysis. Collected cells were lysed with RIPA lysis buffer (1\% NP-40, $0.1 \%$ SDS, $0.5 \%$ sodium deoxycholate, $150 \mathrm{mmol} / \mathrm{l} \mathrm{NaCl}$ and $10 \mathrm{mmol} / \mathrm{l}$ Tris- $\mathrm{HCl}$ ) containing $1 / 100$ phenylmethanesulfonyl fluoride solution. The total protein concentration was measured by using the BCA method (Beyotime Institute of Biotechnology, Haimen, China). Aliquots $(20 \mu \mathrm{g})$ of each sample were separated by $10 \%$ SDS-PAGE (14) and transferred to polyvinylidene fluoride membranes for probing with the following primary antibodies: rabbit anti-human NEDD4 (dilution, 1:1,000; cat. no. ab14592; Abcam, Cambridge, UK), mouse anti-human AKT, rabbit anti-human p-AKT (dilutions, 1:1,000/1:500 and cat nos. sc-5298/135650, respectively from Santa Cruz Biotechnology, Inc., Dallas, TX, USA), rabbit anti-human PTEN (dilution, 1:1,000; cat. no. ab32199; Abcam), mouse anti-human E-cadherin (dilution, 1:1,000; cat. no. ab1416; Abcam), mouse anti-human vimentin (dilution, 1:2,000; cat. no. ab8979; Abcam) and mouse anti-human glyceraldehyde 3-phosphate dehydrogenase (GAPDH; dilution, 1:5,000; cat. no. ab8245, Abcam). Immunoreactive bands were assessed by optical densitometry analysis using the Image J version 1.42 software. Signal values were adjusted using GAPDH as the loading control.

Immunocytochemistry (ICC). Cells were grown on coverslips in 6-well dishes and transfected with NEDD4-siRNA. Subsequent to $48 \mathrm{~h}$, the cells were washed with PBS, fixed with $2 \%(\mathrm{w} / \mathrm{v})$ paraformaldehyde and permeabilized with $1 \%$ (v/v) Triton X-100. Cells were then blocked by incubating with $10 \%(\mathrm{w} / \mathrm{v})$ normal goat serum in PBS at room temperature for $1 \mathrm{~h}$ and were then reacted with one of the primary antibodies by incubation at $4^{\circ} \mathrm{C}$ overnight. The following day, the cells were washed and incubated at $37^{\circ} \mathrm{C}$ for $1 \mathrm{~h}$ with $\mathrm{Cy} 3$-labeled secondary antibody (Beyotime Institute of Biotechnology, Haimen, China) at room temperature, and then co-stained with DAPI (Sigma-Aldrich; Merck Millipore). Double staining with NEDD4 (cat. no. ab14592; Abcam) and PTEN (cat. no. sc7974; Santa Cruz Biotechnology, Inc.) was carried out, and the Cy3-(cat. no. A0516) and Alexa Fluor 488-labeled secondary antibodies (cat. no. A0423) (dilution, 1:400; Beyotime Institute of Biotechnology) were used. Images of the immunostained cells were obtained using a fluorescence microscope (magnification, x200) (14).

Statistical analysis. Statistical analyses were conducted with SPSS software (version 17.0; SPSS, Inc., Chicago, IL, USA). 
Table I. Clinicopathological correlation of NEDD4 expression in patients with hepatocellular carcinoma.

\begin{tabular}{|c|c|c|c|}
\hline Variables & $\mathrm{NEDD}^{+}, \mathrm{n}(\%)$ & $\mathrm{NEDD}^{-}, \mathrm{n}(\%)$ & P-value \\
\hline Age, years & & & 0.826 \\
\hline$>50$ & $32(58.2)$ & $14(60.9)$ & \\
\hline$\leq 50$ & $23(41.8)$ & $9(39.1)$ & \\
\hline Sex & & & 0.670 \\
\hline Male & $38(69.1)$ & $17(73.9)$ & \\
\hline Female & $17(30.9)$ & $6(26.1)$ & \\
\hline Hepatitis & & & 0.922 \\
\hline $\mathrm{HBV}$ & $36(65.5)$ & $16(69.6)$ & \\
\hline $\mathrm{HCV}$ & $10(18.2)$ & $4(17.4)$ & \\
\hline None & $9(16.3)$ & $3(13.0)$ & \\
\hline Alpha fetal protein $(\mathrm{ng} / \mathrm{ml})$ & & & 0.264 \\
\hline$>400$ & $36(65.5)$ & $18(78.3)$ & \\
\hline$\leq 400$ & $19(34.5)$ & $5(21.7)$ & \\
\hline Tumor size (cm) & & & 0.047 \\
\hline$>3$ & $35(63.6)$ & $9(39.1)$ & \\
\hline$\leq 3$ & $20(36.4)$ & $14(60.9)$ & \\
\hline Liver cirrhosis & & & 0.734 \\
\hline Yes & $31(56.4)$ & $12(52.2)$ & \\
\hline No & $24(43.6)$ & $11(47.8)$ & \\
\hline Tumor number & & & 0.367 \\
\hline Single & $18(32.7)$ & $10(43.5)$ & \\
\hline Multiple & $37(67.3)$ & $13(56.5)$ & \\
\hline Degree of differentiation & & & 0.032 \\
\hline Well & $11(20.0)$ & $11(47.8)$ & \\
\hline Moderate & $19(34.5)$ & $7(30.5)$ & \\
\hline Poor & $25(45.5)$ & $5(21.7)$ & \\
\hline Tumor node metastasis stage & & & 0.608 \\
\hline $\mathrm{I}+\mathrm{II}$ & $30(54.5)$ & $14(60.9)$ & \\
\hline III+IV & $25(45.5)$ & $9(39.1)$ & \\
\hline Vascular invasion & & & $<0.001$ \\
\hline Yes & $49(89.1)$ & $11(47.8)$ & \\
\hline No & $6(10.9)$ & $12(52.2)$ & \\
\hline Lymph node metastasis & & & 0.005 \\
\hline Yes & $42(76.4)$ & $10(43.5)$ & \\
\hline No & $13(23.6)$ & $13(56.5)$ & \\
\hline
\end{tabular}

NEDD4, neural precursor cell-expressed developmentally downregulated protein 4.

All quantitative data were calculated as the mean \pm standard deviation using data from at least 3 independent experiments. Statistical evaluation of the data was performed with one-way analysis of variance (Bonferroni method). Pair-wise comparisons were conducted using a Student's t-test. $\mathrm{P}<0.05$ was considered to indicate a statistically significant difference.

\section{Results}

NEDD4 and PTEN protein expression levels in normal hepatocyte and HCC cell lines. Western blot analysis detection of NEDD4 and PTEN expression revealed different levels of the two proteins among the normal liver cell LO2 and HCC cells including Huh7, Hep3B, PLC/PRF/5 and SMMC7721 cell lines. In general, the protein expression of NEDD4 and PTEN were different in normal liver cell compared with all detected HCC cells. According to the results, all detected HCC cells presented higher expression levels of NEDD4 and lower expression levels of PTEN compared with LO2. Since the Hep3B cell line exhibited high expression of NEDD4, it was selected for the subsequent siRNA-mediated knockdown experiments.

Depletion of NEDD4 suppresses the ability of growth and migration in HCC cells. It has been reported that NEDD4 
A

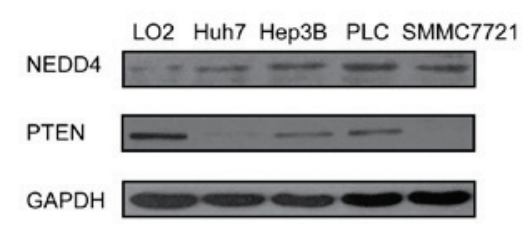

B

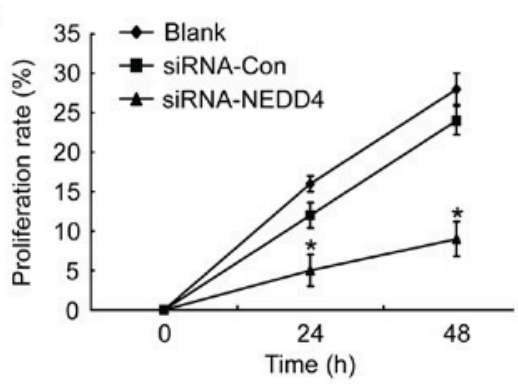

C

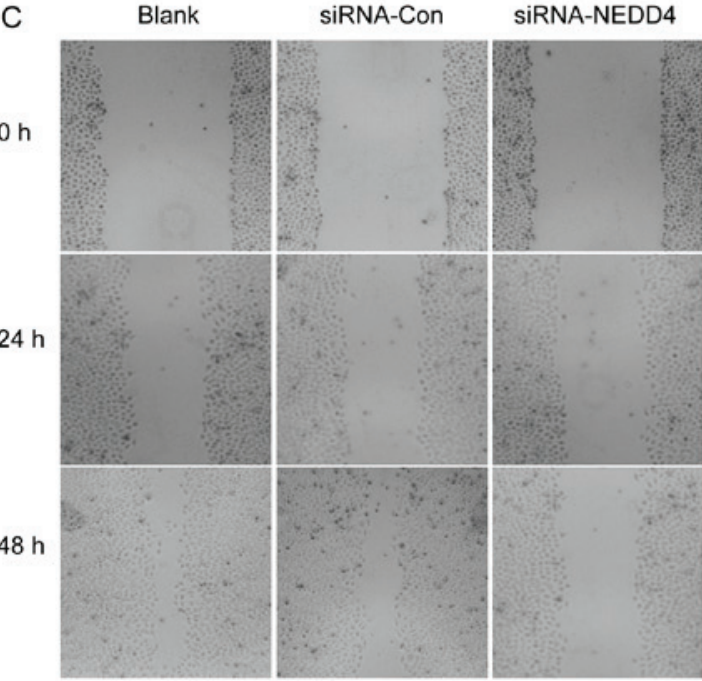

D

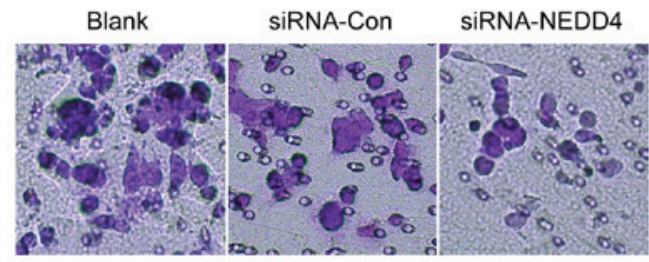

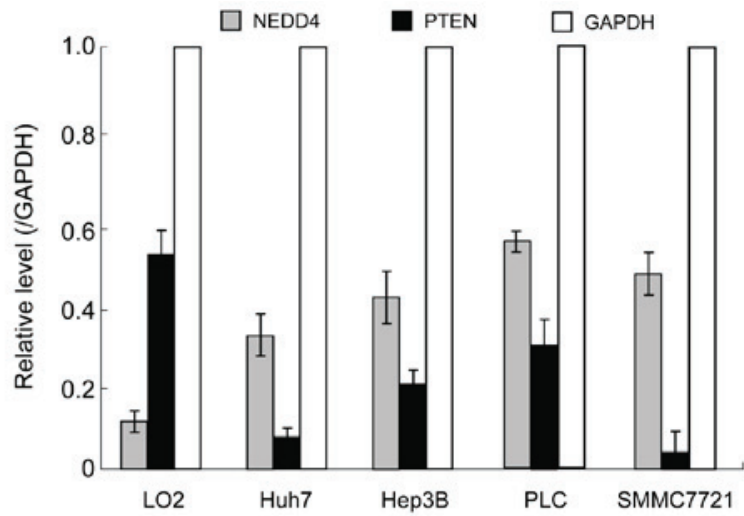
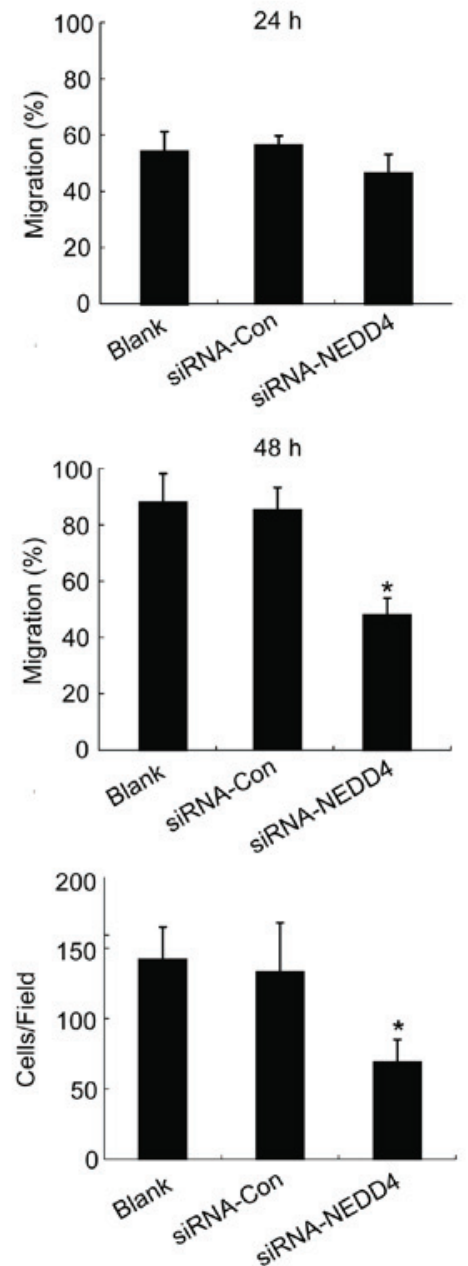

Figure 1. NEDD4 depletion and effects on HCC cellular proliferation, migration and invasion. (A) Western blot analysis detection of NEDD4 and PTEN expression in LO2 and various HCC cell lines. (B) Cellular viability analyzed by an MTT assay. Hep3B cells were pre-treated with NEDD4-siRNA and the proliferation rate was measured at 24 and $48 \mathrm{~h}$. NEDD4 depletion led to a significant inhibition of cell proliferation. (C) Wound-healing assays and (D) Transwell assays revealed the inhibitory effect of NEDD4 depletion on cell migration and invasion ability. "P<0.05, compared with blank control and siRNA-control groups. Data is presented as the mean \pm standard deviation. NEDD4, neural precursor cell-expressed developmentally downregulated protein 4; HCC, hepatocellular carcinoma; PTEN, phosphatase and tensin homolog; LO2, normal liver cells; siRNA, small interfering RNA.

may be an important factor in the malignant transformation processes $(9,10)$. Therefore, the present study investigated whether depletion of NEDD4 would have an impact on HCC cells' ability to grow and migrate. An MTT assay revealed that
NEDD4 siRNA-transfected Hep3B cells exhibited a significantly decreased proliferation rate compared with control blank (no treatment) cells at 24 and $48 \mathrm{~h}$ following transfection ( 15 to $4 \%$ at $24 \mathrm{~h}$ and 28 to $9 \%$ at $48 \mathrm{~h} ; \mathrm{P}<0.05 ;$ Fig. $1 \mathrm{~B}$ ). These 


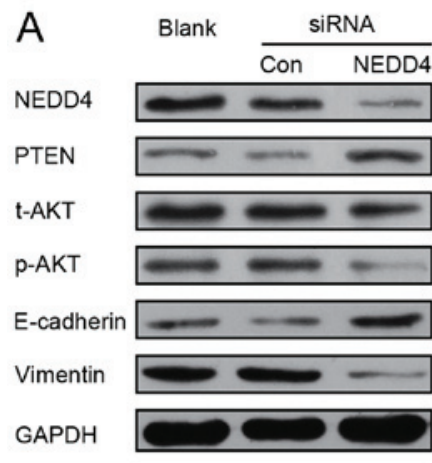

B

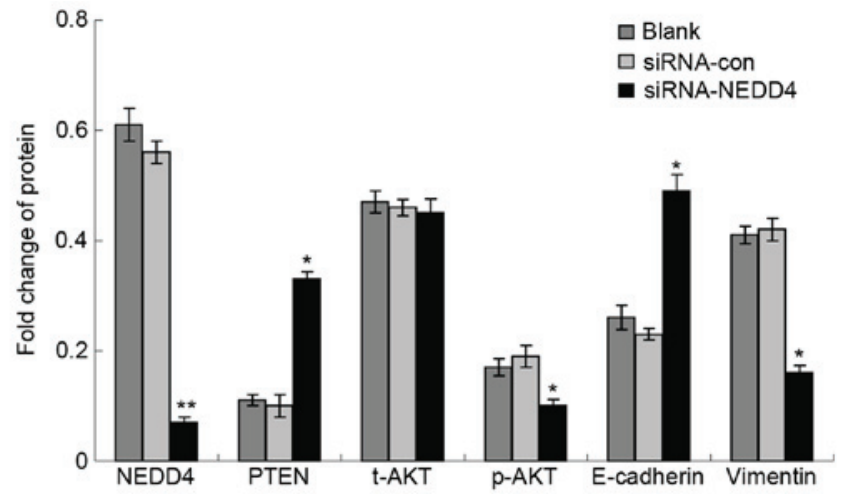

NEDD4

PTEN

Merge

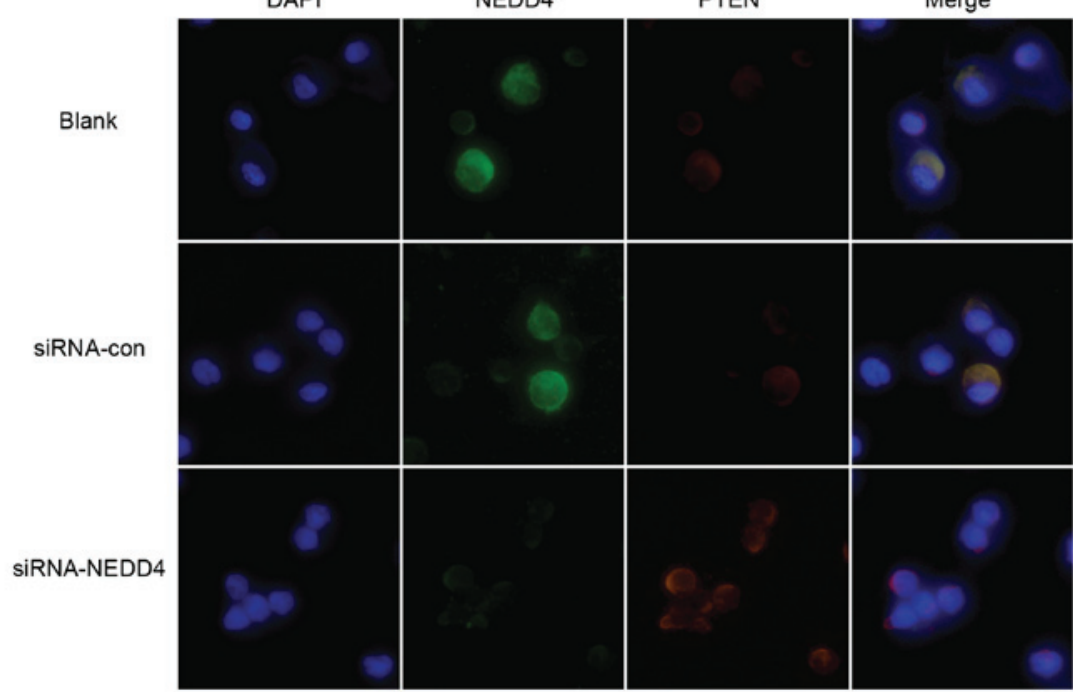

Figure 2. NEDD4 affects the PTEN/PI3K/AKT signaling pathways in HCC cells. (A) Western blot analysis demonstrating the effect of siRNA depletion of NEDD4. NEDD4-depleted HCC cells demonstrated increased expression of PTEN and decreased phosphorylation level of AKT. EMT markers (E-cadherin and vimentin) were detected simultaneously. (B) Immunofluorescence detection of NEDD4 and PTEN showing that depletion of NEDD4 negatively affected the expression of PTEN in Hep3B cells. ${ }^{*} \mathrm{P}<0.05$ and ${ }^{* *} \mathrm{P}<0.01$ compared with blank control and siRNA-control groups. Data is presented as the mean \pm standard deviation. NEDD4, neural precursor cell-expressed developmentally downregulated protein 4; PTEN, phosphatase and tensin homolog; PI3K, phosphoinositide 3-kinase; AKT, protein kinase B; HCC, hepatocellular carcinoma; siRNA, small interfering RNA; EMT, epithelial-mesenchymal transition.

results suggest that depletion of NEDD4 negatively influences cell proliferation in HCC cells.

In order to study the migration potential of NEDD4-depleted HCC cells, and thus the NEDD4 role in HCC cell metastasis, wound-healing experiments were performed. Results at $48 \mathrm{~h}$ demonstrated a statistically significant decrease in migration ability for the NEDD4-siRNA transfected HCC cells compared with the blank control cells (45 vs. $88 \%$, respectively; $\mathrm{P}<0.05$; Fig. 1C). However, no significant differences were observed at $24 \mathrm{~h}$. The present study also conducted Transwell assays with matrigel in order to investigate whether NEDD4 is capable of promoting the invasive capacity of HCC cells and whether NEDD4 depletion leads to decreased invasion. Results from the invasion assay demonstrated significantly decreased invasiveness for the NEDD4-siRNA cells compared with the blank control and siRNA-control groups $(\mathrm{P}<0.05$; Fig. 1D). Thus, depletion of NEDD4 decreased the HCC cells' capacities for proliferation, migration and invasion, all of which are key features of tumorigenesis and tumor development.

Furthermore, the present study studied the impact of NEDD4 depletion on PTEN and the PI3K/AKT signaling pathway in HCC cells. The NEDD4-siRNA transfected cells demonstrated a significant reduction of NEDD4 protein expression, compared with the blank control and siRNA-control groups (Fig. 2). Depletion of NEDD4 increased the expression of PTEN, and had no effect on the expression level of total AKT but reduced the expression of p-AKT (Fig. 3). Immunofluorescence experiments confirmed these results (Fig. 3).

Depletion of NEDD4 affects epithelial-mesenchymal transition $(E M T)$. To investigate whether the expression level of NEDD4 has an impact on EMT, the present study measured EMT markers, including the epithelial marker E-cadherin and the mesenchymal marker vimentin, in HCC and NEDD4-depleted HCC cells. As expected, the EMT marker vimentin was clearly expressed in tumor cells. Notably, NEDD4-depleted HCC cells exhibited increased E-cadherin and decreased vimentin protein levels (Fig. 3) Immunofluorescence experiments confirmed these results (Fig. 3).

Overexpression of NEDD4 in HCC correlates with tumor size, differentiation degree, vascular invasion and lymph node metastasis. NEDD4 expression was evaluated in paraffin-embedded serial sections of cancerous and non-cancerous tissues from 78 patients with HCC. On average, the HCC tissues exhibited higher expression of NEDD4, lower 


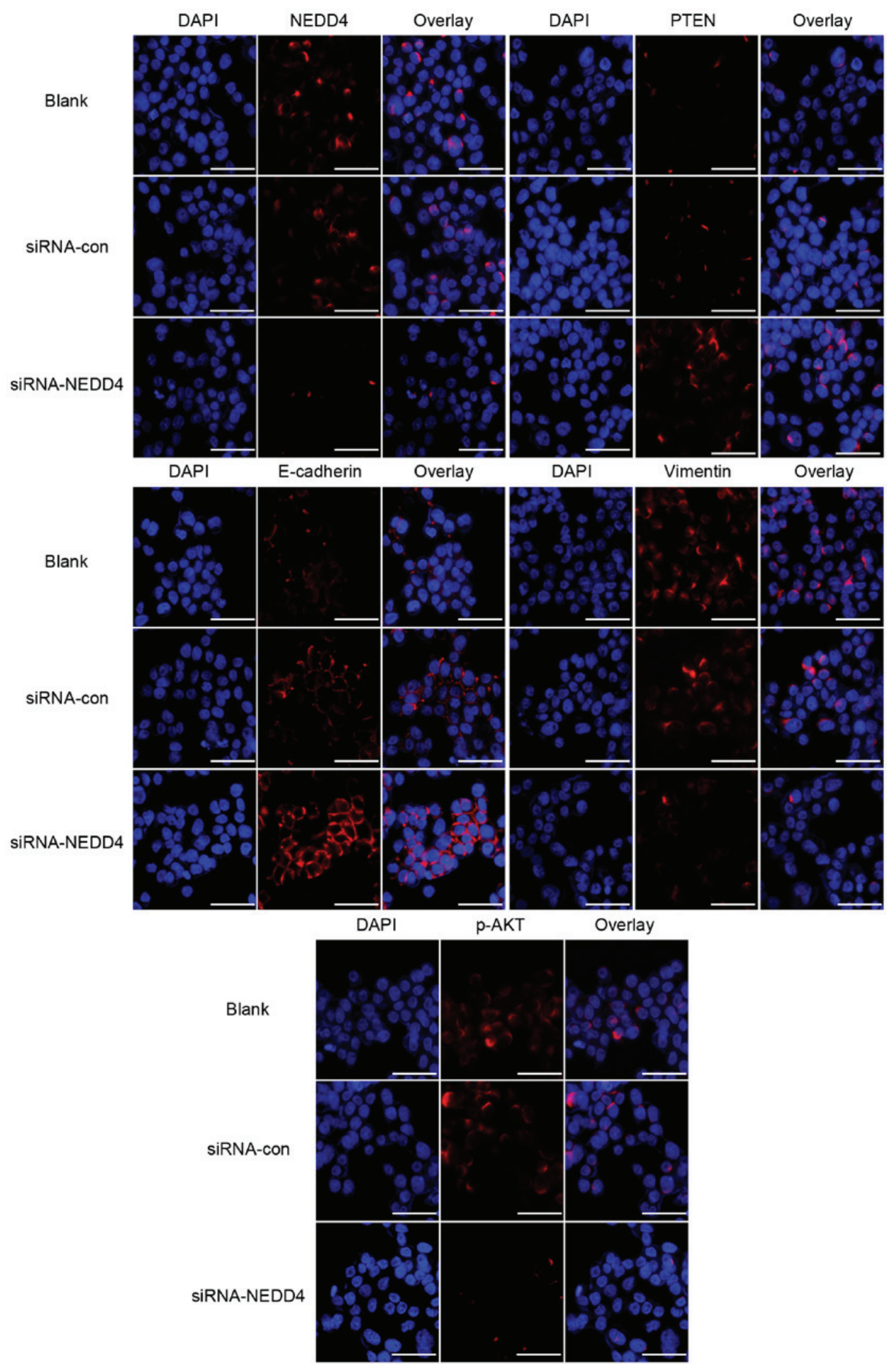

Figure 3. NEDD4 effects HCC cells by regulating PTEN/PI3K/AKT signaling pathways. NEDD4, PTEN and p-AKT were detected by immunofluorescence assay in addition to the epithelial-mesenchymal-transition markers E-cadherin and vimentin. The results revealed that depletion of NEDD4 was negatively associated with PTEN expression and phosphorylation level of AKT. Gain of E-cadherin expression and loss of vimentin expression is shown. Scale bar, $50 \mu \mathrm{m}$. NEDD4, neural precursor cell-expressed developmentally downregulated protein 4; HCC, hepatocellular carcinoma; PTEN, phosphatase and tensin homolog; PI3K, phosphoinositide 3-kinase; AKT, protein kinase B; p-, phosphorylated; siRNA, small interfering RNA.

expression of PTEN and higher expression of p-AKT compared with the adjacent non-tumor liver tissues (Fig. 4). NEDD4 overexpression was identified in $70.5 \%(55 / 78)$ of the patients with HCC. Immunohistochemically, NEDD4 was principally 


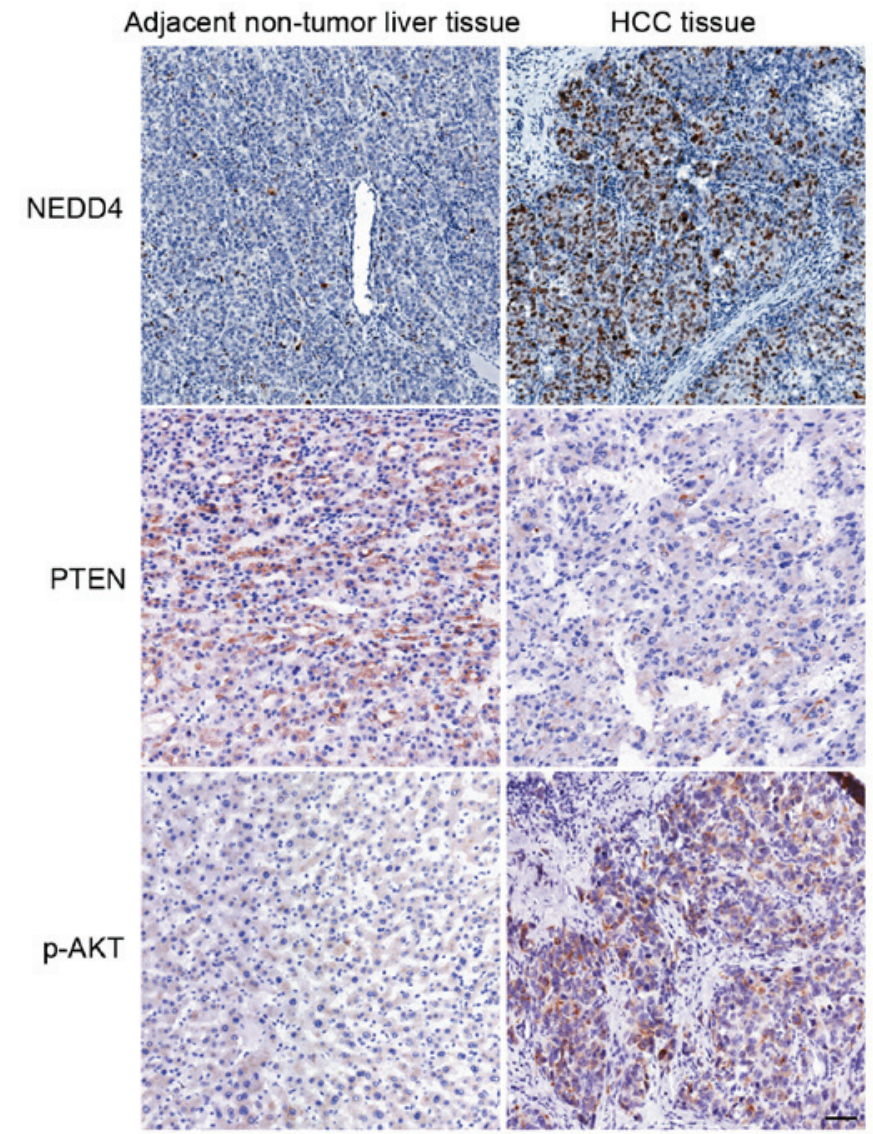

Figure 4. Representative immunohistochemistry images of human HCC tissue specimens immunostained for NEDD4, PTEN and p-AKT. Magnification, x200. Scale bar, $50 \mu \mathrm{m}$. HCC, hepatocellular carcinoma; NEDD4, neura precursor cell-expressed developmentally downregulated protein 4; PTEN, phosphatase and tensin homolog; p-AKP, phosphorylated AKT.

detected in the cytoplasm and nucleus of tumor cells. Table I summarizes the association of immunohistochemical NEDD4 expression with the various clinicopathological parameters of the patients. The expression of NEDD4 was significantly associated with tumor size $(\mathrm{P}=0.032)$, vascular invasion $(\mathrm{P}<0.001)$, differentiation grade $(\mathrm{P}=0.032)$ and lymph node status $(\mathrm{P}=0.005)$. These observations suggest that NEDD4 expression level may be a predictive factor for the recurrence and survival rate of patients with HCC.

\section{Discussion}

HCC was ranked as the third most lethal type of cancer in $2008(15,16)$. The mortality rate of HCC is almost identical to its rate of incidence, a fact that underlies the high fatality rate of HCC (17-19). In Asia, incidence rates of HCC are continuing to rise and its level is considered to be nearing an epidemic proportion (20). Early resectable presentation is rare (only $10-20 \%$ of cases) since the disease is clinically silent at this stage. The outcomes for advanced, inoperable stages are unsatisfactory and treatments are typically limited to palliation (21).

The molecular events of pathogenesis, tumorigenesis and metastasis in HCC are still poorly understood. The HCC tumor markers that have been developed for clinical use include: a-fetoprotein, squamous cell carcinoma antigen, golgiprotein73 or des- $\gamma$-carboxy prothrombin, however these have limited sensitivity and specificity $(22,23)$. Identification of other diagnostic and prognostic biomarkers (and consequent therapeutic targets) is imperative to improve detection of $\mathrm{HCC}$ as well as treatment and follow-up of patients. To the best of our knowledge, there are limited studies on the relevance of NEDD4 to the progression of liver cancer and the molecular mechanisms underlying the association of NEDD4 with liver cancer remain unclear. The present data show that NEDD4 overexpression in $\mathrm{HCC}$ is associated with the processes of tumorigenesis and tumor progression, which would support a poor prognosis and reduced overall survival rate. Results from HCC cell lines (vs. normal hepatocytes) and tumor specimens from patients with HCC (vs. adjacent non-tumor liver tissue) confirm that overexpression of NEDD4 is associated with the tumor microenvironment. Furthermore, the present data suggest that NEDD4 depletion affects the phosphorylation of AKT, which supports the hypothesis that NEDD4 is a potential oncoprotein and a promoting factor of the PTEN/PI3K/AKT signaling pathway. In addition, NEDD4 depletion in HCC cells may allow PTEN to negatively regulate the PI3K signaling and exert its functions as a tumor suppressor protein, thereby inhibiting an overactive PTEN/PI3K/AKT pathway cascade. NEDD4 transcription is known to be positively regulated by the PI3K pathway, representing a positive feedback for PTEN degradation and PI3K activation (12). Therefore, the effects of NEDD4 depletion on PTEN/PI3K/AKT in HCC cells may have resulted from this upstream and feedback regulatory process.

Statistical analysis of the immunohistochemical staining data and the HCC tumor clinicopathological data indicated a significant correlation of NEDD4 positivity with various HCC characteristics, including tumor size, differentiation degree, vascular invasion and lymph node status. These observations suggest that NEDD4 expression level could be a predictive factor for recurrence and survival rate of patients with HCC. In addition, NEDD4 overexpression was evidently linked to HCC metastasis, and thus to a poor prognosis. It may be speculated that patients with a NEDD4-negative HCC status would have improved prognosis, therapy response and survival rate. If this assumption could be proven true, NEDD4 would be an effective predictor for post-surgery survival rate of HCC.

Knockdown experiments in the present study revealed that depletion of NEDD4 in HCC cells markedly reduced cellular migration and invasion, the two key cellular processes in metastasis, and also impacted cellular proliferation. These results may translate into clinical practice, as they suggest that NEDD4 is positively associated with HCC metastatic potential and higher TNM staging, and thus hint at its uses as a predictive biomarker of the poor prognosis of HCC.

NEDD4 is homologous to E6-AP carboxyl terminus E3 ubiquitin ligase, which is involved in ubiquitination of the tumor suppressor gene PTEN to cause its proteasomal degradation and nuclear translocation. It has thus been suggested that NEDD4 may have a pro-oncogenic role (10,24-27). In agreement with this pathological function, overexpression of NEDD4 has been observed in various types of cancer, including gastric (24), lung (23) and colorectal (10). Studies of NEDD4 in colorectal cancer have demonstrated its role in promoting tumor cell growth (10). However, Eide et al (13) previously demonstrated that the overexpression of NEDD4-1 
in colon cancer tissues did not correlate with the downregulation of PTEN. This seemingly contradictory finding may actually suggest that in certain tumor entities NEDD4 may support oncogenesis and tumor progression independently of the downregulation of PTEN. In non-small cell lung cancer (NSCLC), inhibition of NEDD4 expression significantly suppresses proliferation of NSCLC cells and tumor growth, as shown by in vivo studies $(24,28)$. Furthermore, NEDD4 overexpression was shown to augment the tumorigenicity of lung cancer cells, while producing no impact on PTEN gene expression (24). Recently, Liao et al (27) reported that NEDD4 is associated with cancer metastasis through its regulation of the processes underlying tumor invasion, apoptosis and colonization.

To the best of our knowledge, the present study revealed that NEDD4 promotes HCC cell invasion and migration, which is tightly associated with HCC metastasis. The present data indicate that NEDD4 may be an exceptional biomarker for predicting the poor prognosis of post-surgery HCC. Translating these finding into clinical practice may embody the detection of NEDD4 overexpression in HCC tumors by immunohistochemistry techniques and the results may be used to guide the treatment approach (i.e., tailoring the treatment to NEDD4-positive or -negative status). Additionally, NEDD4 is suggested as a novel therapeutic target, due to its positive correlation with the invasion and migration capacities of the HCC cell lines. Depletion or inhibition of NEDD4 may be a strategic approach to reduce metastasis and delay tumor recurrence, thereby improving survival rate. Further studies, focusing in particular on the correlation of NEDD4 expression and overall survival rate, should be carried out on larger populations of patients with HCC.

\section{Acknowledgements}

The authors would like to thank Professor L. Dagna for his intellectual and critical advice on the present study.

\section{References}

1. Siegel R, Naishadham D and Jemal A: Cancer statistics, 2013 CA Cancer J Clin 63: 11-30, 2013.

2. Hanahan D and Weinberg RA: Hallmarks of cancer: The next generation. Cell 144: 646-674, 2011.

3. Farazi PA and DePinho RA: Hepatocellular carcinoma pathogenesis: From genes to environment. Nat Rev Cancer 6: 674-687, 2006.

4. Aravalli RN, Steer CJ and Cressman EN: Molecular mechanisms of hepatocellular carcinoma. Hepatology 48: 2047-2063, 2008.

5. Tang ZY, Ye SL, Liu YK, Qin LX, Sun HC, Ye QH, Wang L, Zhou J, Qiu SJ, Li Y, et al: A decade's studies on metastasis of hepatocellular carcinoma. J Cancer Res Clin Oncol 130: 187-196, 2004

6. Donovan P and Poronnik P: Nedd4 and Nedd4-2: Ubiquitin ligases at work in the neuron. Int J Biochem Cell Biol 45: 706-710, 2013.

7. Kerscher O, Felberbaum R and Hochstrasser M: Modification of proteins by ubiquitin and ubiquitin-like proteins. Annu Rev Cell Dev Biol 22: 159-180, 2006.
8. Kumar S, Tomooka Y and Noda M: Identification of a set of genes with developmentally down-regulated expression in the mouse brain. Biochem Biophys Res Commun 185: 1155-1161, 1992.

9. Nakayama KI and Nakayama K: Ubiquitin ligases: Cell-cycle control and cancer. Nat Rev Cancer 6: 369-381, 2006.

10. Chen C and Matesic LE: The Nedd4-like family of E3 ubiquitin ligases and cancer. Cancer Metastasis Rev 26: 587-604, 2007.

11. BelletMM,Piobbico D,BartoliD,Castelli M,Pieroni S,BrunacciC, Chiacchiaretta M, Del Sordo R, Fallarino F, Sidoni A, et al: NEDD4 controls the expression of GUCD1, a protein upregulated in proliferating liver cells. Cell Cycle 13: 1902-1911, 2014.

12. Baker SJ: PTEN enters the nuclear age. Cell 128: 25-28, 2007.

13. Eide PW, Cekaite L, Danielsen SA, Eilertsen IA, Kjenseth A, Fykerud TA, Ågesen TH, Bruun J, Rivedal E, Lothe RA and Leithe E: NEDD4 is overexpressed in colorectal cancer and promotes colonic cell growth independently of the PI3K/PTEN/AKT pathway. Cell Signal 25: 12-18, 2013.

14. Bao L, Yan Y, Xu C, Ji W, Shen S, Xu G, Zeng Y, Sun B, Qian H, Chen L, et al: MicroRNA-21 suppresses PTEN and hSulf-1 expression and promotes hepatocellular carcinoma progression through AKT/ERK pathways, Cancer Lett 337: 226-236, 2013.

15. Llovet JM, Burroughs A and Bruix J: Hepatocellular carcinoma. Lancet 362: 1907-1917, 2003.

16. Venook AP, Papandreou C, Furuse J and de Guevara LL: The incidence and epidemiology of hepatocellular carcinoma: A global and regional perspective. Oncologist 15 (Suppl 4): 5-13, 2010.

17. Bosch FX, Ribes J, Díaz M and Cléries R: Primary liver cancer: Worldwide incidence and trends. Gastroenterology 127 (5 Suppl 1): 5-16, 2004.

18. Forner A, Llovet JM and Bruix J: Hepatocellular carcinoma. Lancet 379: 1245-1255, 2012.

19. Yuen MF, Hou JL and Chutaputti A; Asia Pacific Working Party on Prevention of Hepatocellular Carcinoma: Hepatocellular carcinoma in the Asia pacific region. J Gastroenterol Hepatol 24: 346-353, 2009.

20. Mann CD, Neal CP, Garcea G, Manson MM, Dennison AR and Berry DP: Prognostic molecular markers in hepatocellular carcinoma: A systematic review. Eur J Cancer 43: 979-992, 2007.

21. Okano $H$, Nakajima $H$, Tochio $T$, Suga D, Kumazawa $H$, Isono $\mathrm{Y}$, Tanaka $\mathrm{H}$, Matsusaki S, Sase T, Saito T, et al: A case of a resectable single hepatic epithelioid hemangioendothelioma with characteristic imaging by ADC map. Clin J Gastroentero 8: 406-413, 2015.

22. Zhao YJ, Ju Q and Li GC: Tumor markers for hepatocellular carcinoma. Mol Clin Oncol 1: 593-598, 2013.

23. Sakata T, Sakaguchi H, Tsuda L, Higashitani A, Aigaki T, Matsuno K and Hayashi S: Drosophila Nedd4 regulates endocytosis of notch and suppresses its ligand-independent activation. Curr Biol 14: 2228-2236, 2004.

24. Amodio N, Scrima M, Palaia L, Salman AN, Quintiero A, Franco R, Botti G, Pirozzi P, Rocco G, De Rosa N and Viglietto G: Oncogenic role of the E3 ubiquitin ligase NEDD4-1, a PTEN negative regulator, in non-small-cell lung carcinomas. Am J Pathol 177: 2622-2634, 2010.

25. Gao C, Pang L, Ren C and Ma T: Decreased expression of Nedd4L correlates with poor prognosis in gastric cancer patient. Med Oncol 29: 1733-1738, 2012.

26. He S, Deng J, Li G, Wang B, Cao Y and Tu Y: Down-regulation of Nedd $4 \mathrm{~L}$ is associated with the aggressive progression and worse prognosis of malignant glioma. Jpn J Clin Oncol 42: 196-201, 2012.

27. Liao CJ, Chi HC, Tsai CY, Chen CD, Wu SM, Tseng YH, Lin YH, Chung IH, Chen CY, Lin SL, et al: A novel small-form NEDD4 regulates cell invasiveness and apoptosis to promote tumor metastasis. Oncotarget 6: 9341-9354, 2015.

28. Sakashita H, Inoue H, Akamine S, Ishida T, Inase N, Shirao K, Mori $\mathrm{M}$ and Mimori K: Identification of the NEDD4L gene as a prognostic marker by integrated microarray analysis of copy number and gene expression profiling in non-small cell lung cancer. Ann Surg Oncol 20 (Suppl 3): 590-598, 2013. 